\title{
Bioanalysis
}

\section{Simultaneous quantitation of the BACE1 inhibitor AZD3293 and its metabolite AZ13569724 in human matrices by LC-MS/ MS}

\begin{abstract}
Aim: AZD3293 is a novel BACE1 inhibitor in Phase III development for Alzheimer's disease. Sensitive and robust bioanalytical methods were required to quantitate AZD3293 and its metabolite AZ13569724 in human biological matrices. Methodology/ results: Human plasma was prepared by protein precipitation. Linearity for both analytes was in the range of $0.5-500 \mathrm{ng} / \mathrm{ml}$ with up to 100 -fold dilution. Plasma ultrafiltrate samples were prepared using Centrifree ${ }^{\circledR}$ ultrafiltration device. Urine and CSF samples were analyzed directly after dilution. A 27\% decrease in AZD3293 concentrations in the CSF collection apparati was found due to nonspecific binding. Incurred sample reanalysis was acceptable. Conclusion: Methods for simultaneous quantitation of AZD3293 and its metabolite AZ13569724 in human biological matrices have been validated and successfully applied to clinical studies.
\end{abstract}

First draft submitted: 11 January 2017; Accepted for publication: 27 March 2017; Published online: 24 April 2017

Keywords: AZD3293 • BACE1 • cerebrospinal fluid • LC-MS/MS • plasma • PUF • urine

Amyloid-lowering therapies for the treatment of Alzheimer's disease (AD) have been a primary research focus for many years, with BACE1 inhibition being one of the most attractive approaches [1]. Amyloid pathology is one of the major hallmarks of pathology in AD [2,3]. The cleavage of APP by BACE is the first step in the production of $A \beta$ and is considered the most promising target for reducing amyloid pathology [4]. To test this hypothesis, several generations of BACE1 inhibitor drug candidates have been developed with incremental improvements, however, this hypothesis is still unproven in humans [1].

AZD3293 is a novel, potent, brain-permeable BACE 1 inhibitor [5]. It is being developed as a potentially disease-modifying treatment for $\mathrm{AD}$ in patients diagnosed with early $\mathrm{AD}$, defined as the continuum of patients with mild cognitive impairment due to $\mathrm{AD}$ and patients diagnosed with mild dementia of the Alzheimer's type. The efficacy and safety of AZD3293 as a treatment for early $\mathrm{AD}$ are currently being evaluated in two global Phase III studies (AMARANTH, NCT02245737; and DAYBREAK-ALZ, NCT02783573).

Accurate quantitative determination of drugs and metabolites in a physiological matrix is critical to drug development. Robust bioanalytical methods help researchers to develop a thorough understanding of pharmacokinetics (PK) across a variety of doses and/or regimens, which in turn enables the determination of absorption, distribution, metabolism and elimination dynamics for investigational drugs. Guidance for the characterization and validation of bioanalytical methods for use in nonclinical and clinical studies of investigational drug products has been provided by a number of regulatory agencies including the EMA [6] and the US FDA [7,8]. LC-MS/MS has been proven to be highly selective and sensitive, and is a widely used methodology for the quantitation of drugs used in clinical practice for $\mathrm{AD}$ in a wide range of biological
Yan Li*,1, Paul H Severin², Mark R Hoffmann², Dennis L Miller ${ }^{2}$, Scott A Monk ${ }^{3}$

\section{\& Alan R Kugler 4}

'Early Clinical Development, IMED Biotech Unit, AstraZeneca, Waltham, MA, USA 02451

${ }^{2}$ Clinical Laboratories, Covance Laboratories, Inc., Madison, WI, USA 53704

${ }^{3}$ Drug Disposition, Lilly Research Laboratories, Eli Lilly \& Company, Indianapolis, IN, USA 46285

${ }^{4}$ Clinical Pharmacology, Coastal Pharma Group, Concord, MA, USA 01742

*Author for correspondence:

Tel.: +1 2153139672

Fax: +1 7814725927

yan.li@astrazeneca.com 
matrices [9]. In addition, recent guidance on safety testing of drug metabolites $[10,11]$ also recommends the assessment of exposure in toxicology studies for any metabolite accounting for $>10 \%$ of exposure to total drug related material in humans. Human biological matrices typically include plasma and urine for determining the level of systemic exposure and information about metabolism and potential routes of elimination. In addition, plasma ultrafiltrate (PUF) is sometimes used for determining drug plasma protein binding, which enables the determination of free drug concentrations. In the case of drugs targeted for the treatment of neurological diseases, it is essential that a drug candidate is brain-permeable and reaches pharmacodynamically active drug concentrations at the putative target. While there are a number of methods available to directly quantify brain concentrations in nonclinical studies, the only method readily available in clinical studies to serve as a surrogate marker of brain drug concentrations is measuring drug concentrations in samples of cerebrospinal fluid (CSF) [12]. The resulting PK parameters for CSF drug concentrations, along with biomarker or pharmacodynamic (PD) response data, enable the modeling of PK/PD relationships. Such models are critical to understanding exposure response and the corresponding therapeutic index for the drug (i.e., relationship between pharmacologic response and safety/tolerability across doses).

In the interest of scientific transparency, this paper is the first published report of the bioanalytical methods developed and validated in support of the AZD3293 clinical program to simultaneously quantitate AZD3293 (Figure 1) and its major metabolite AZ13569724 in human plasma, urine, PUF, and CSF. We also report the results of additional evaluations to further investigate the nonspecific binding potentials of both analytes in the CSF collection apparati. In preclinical toxicology studies of AZD3293, the major circulating metabolite was identified as AZ13569724. Safety pharmacology studies of AZ13569724 demonstrated that it has no significant effect on cardiac ion channels (including hERG) or on 150 other secondary pharmacology targets explored (EKETJALl SA ET AL., UNPUBLISHED DATA). To determine if concentrations of AZ13569724 were sufficient to potentially contribute to the $\mathrm{PD}$ response observed with AZD3293, and to properly investigate the PK of this metabolite per guidelines on safety testing of drug metabolites $[10,11]$, analytical methods were developed to simultaneously quantify AZD3293 and AZ13569724 in human biological matrices. These methods were developed by Covance Laboratories, Inc. (WI, USA), under the Covance-AstraZeneca Clinical Bioanalysis Alliance (WI, USA and MA, USA), and were used for PK characterizations in numerous Phase I clinical pharmacology studies in the USA and Japan, includ- ing single-dose and multiple-dose safety/tolerability/ PK studies in healthy adult subjects and patients with $\mathrm{AD}$ [13] (in which report concentration-time profiles for both analytes are presented), a preliminary assessment of drug-drug interactions, a thorough study of effects on QT prolongation, a mass-balance human ADME study and a study of the relative bioavailability of different oral tablet formulations compared with an oral solution formulation Furthermore, the bioanalytical methods for plasma and CSF are being used in the ongoing Phase III studies and will be used in numerous additional ongoing or planned clinical pharmacology studies to support product registration and effective use. All clinical studies of AZD3293 from which clinical samples were obtained for analysis with these methods were approved by independent Institutional Review Boards and were performed in accordance with the Declaration of Helsinki of 1975 and International Conference on Harmonization/Good Clinical Practice guidelines.

\section{Methods}

\section{Materials, matrices \& reagents}

AZD3293 (also known as LY3314814; IUPAC name: [1 $r, 1^{\prime} R, 4 R$ )-4-methoxy-5"'-methyl-6'-[5-(prop-1yn-1-yl)pyridin-3-yl]-3' $H$-dispiro [cyclohexane1,2'-indene-1',2"-imidazol]-4"'-amine]) and its M1 metabolite (AZ13569724) were supplied by AstraZeneca (Södertälje, Sweden). Isotope-labelled internal standards (ISTDs) $\left[{ }^{13} \mathrm{CD}_{3}\right]-\mathrm{AZD} 3293$ and $\left[{ }^{13} \mathrm{CD}_{3}\right]$-AZ13569724 were supplied by AstraZeneca (Södertälje, Sweden or Alderley Park, UK).

Human plasma ( $\mathrm{K}_{2}$ EDTA anticoagulant), human urine, human PUF and human CSF for spiking were sourced from BioreclamationIVT (NY, USA). Acetonitrile (HPLC grade) and formic acid (96\%), were sourced from Sigma-Aldrich (MO, USA). Acetic acid and Ammonium acetate (Certified ACS) were sourced from Fisher (MA, USA). 3-[(3-Cholamidopropyl) dimethylammonio]-1-propanesulfonate hydrate (CHAPS) was sourced from A.G. Scientific (CA, USA). Water, Type 1, Barnstead system was generated within the bioanalysis laboratory.

\section{Instrumentation}

The method described here is the plasma method. There were some small differences between this and the methods for other matrices, for example, dwell times (CSF only), injection volumes and extraction (protein precipitation for plasma and dilution for the other matrices), depending on the matrix requirements, but overall the methods were comparable.

The LC system used was a Shimadzu LC-20AD or equivalent with integral autosampler and column oven (Shimadzu, Tokyo, Japan). A Supelco Ascentis 
Express C18 $50 \times 3 \mathrm{~mm}, 2.7 \mu \mathrm{m}$ particle size analytical column was used and maintained at $30^{\circ} \mathrm{C}$. A gradient mobile phase system was used with $10 \mathrm{mM}$ ammonium acetate in water:ACN (95:5, v:v) with $0.03 \%$ acetic acid (MA1) or $10 \mathrm{mM}$ ammonium acetate in ACN:water (85:15, v:v) with $0.03 \%$ acetic acid (MA2) with a flow rate of $0.700 \mathrm{ml} / \mathrm{min}$ and the gradient running from $28 \% \mathrm{~B}$ to $56 \% \mathrm{~B}$ over $2.5 \mathrm{~min}$ then increasing to $100 \% \mathrm{~B}$ over $0.1 \mathrm{~min}$ and held for $0.5 \mathrm{~min}$ before re-equilibration. Samples were stored in the autosampler tray under refrigerated conditions and a $10 \mu \mathrm{l}$ sample injection volume was used. The mass spectrometer used was an atmospheric pressure ionization (API) 5000 triple quadrupole mass spectrometer (AB Sciex, CA, USA). This was run in positive electrospray, multiple reaction monitoring, with an ionspray voltage of $5500 \mathrm{~V}$ and Turbolon Spray temperature of $650^{\circ} \mathrm{C}$. In all matrices, the following typical transitions were monitored for the analytes: AZD3293 413.3/323.4 and AZ13569724 399.3/337.3. Typically, a collision energy of $42 \mathrm{eV}$ was used for AZD3293 and AZ13569724.

The labeled internal standards typically used equivalent conditions to the unlabeled compound, except that the dwell time was $60 \mathrm{~ms}$ for AZ13569724 and $40 \mathrm{~ms}$ for the internal standards, and the equivalent carbon-13 and deuterium transitions were analyzed.

\section{Stock solutions, calibration standard \& QC sample preparation}

Stock solutions for individual analytes were prepared by dissolving accurately weighed volumes (corrected for purity, moisture and salt form where appropriate) of AZD3293 and AZ13569724 in ACN:water (30:70, $\mathrm{v}: \mathrm{v})$ with $0.1 \%$ formic acid to achieve a final concentration of $2 \mathrm{mg} / \mathrm{ml}$ for AZD3293 and AZ13569724. These stock solutions were stable for at least 98 and 45 days, respectively, at nominal $4^{\circ} \mathrm{C}$. The internal standards were prepared at $200 \mu \mathrm{g} / \mathrm{ml}$ in the same solvents.

Intermediate solutions were prepared containing all the required analytes and stored in a refrigerator (up to the demonstrated stability). These solutions were used to spike the individual quality control (QC) and calibration levels into the relevant matrix.

The calibration standards for the various human biological matrices tested are shown in Table 1. Prior to establishing frozen matrix stability, fresh calibration standards pools were prepared daily. Once frozen matrix stability was established, either fresh or frozen calibration standards pool could be used. Aliquots of QC samples were stored in a freezer set to maintain a temperature consistent with study specific protocol instructions.

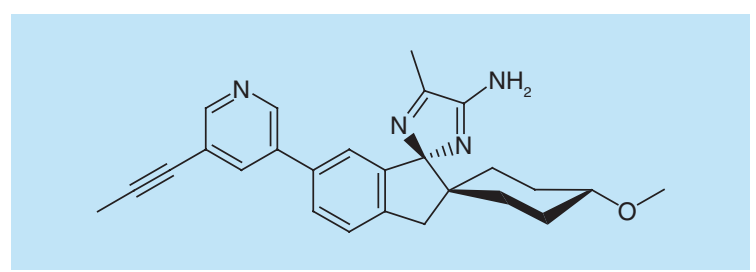

Figure 1. Chemical structure of AZD3293.

\section{Sample preparation \& extraction}

Where possible, automated liquid handling systems were used to aid in the sample preparation. Plasma containing $\mathrm{K}_{2}$ EDTA was used as the control matrix for the analysis. In the clinic, plasma samples were obtained by drawing blood samples into $\mathrm{K}_{2}$ EDTA tubes and then separating the plasma by centrifugation within $30 \mathrm{~min}$ and transferring it to cryovials before freezing. To $50 \mu \mathrm{l}$ plasma sample, $25 \mu \mathrm{l}$ ISTD solution $(50 \mathrm{ng} / \mathrm{ml}$ of AZD3293 and AZ13569724) was added, followed by the addition of $300 \mu \mathrm{l}$ of $0.1 \%$ formic acid ACN. After mixing and centrifugation, the extract was evaporated to dryness and the residue was reconstituted into $150 \mu \mathrm{l} \mathrm{ACN}$ :water (30:70, v:v) with $0.1 \%$ formic acid and the resulting solution was kept refrigerated prior to LC-MS/MS analysis.

Due to the potential for nonspecific binding of the analytes in nonplasma matrices, $0.05 \mathrm{ml}$ of CHAPS $(0.2$ $\mathrm{M})$ was added to $5 \mathrm{ml}$ urine aliquot directly after collection in the clinic prior to freezing. Same procedure was applied to the control matrix as well. To $50 \mu \mathrm{l}$ urine samples, $25 \mu \mathrm{l}$ ISTD solution $(50 \mathrm{ng} / \mathrm{ml}$ of AZD3293 and AZ13569724) was added, followed by the addition of $300 \mu \mathrm{l}$ of $0.1 \%$ formic acid ACN. After mixing and centrifugation, $200 \mu \mathrm{l}$ of extract was evaporated to dryness and the residue was reconstituted into $150 \mu \mathrm{l} \mathrm{ACN}$ :water (30:70, v:v) with $0.1 \%$ formic acid and the resulting solution was kept refrigerated prior to LC-MS/MS analysis.

PUF was prepared from $\mathrm{K}_{2}$ EDTA plasma clinical samples (blank prepared PUF was sourced commercially) in Covance bioanalytical lab. Samples were prepared using a Centrifree ${ }^{\circledR}$ centrifugal ultrafiltration device with a $30 \mathrm{KDa}$ molecular weight cut-off. CHAPS (0.2 $\mathrm{M}, 5 \mu \mathrm{l})$ was added to $250 \mu \mathrm{l}$ plasma in each vial prior to transferring samples to the ultrafiltration device. The sample was mixed well and transferred to the Centrifree centrifugal ultrafiltration device. The PUF was obtained after centrifugation at $3600 \mathrm{rpm}$ for $15 \mathrm{~min}$ at room temperature. To the $75 \mu \mathrm{l}$ PUF sample, $25 \mu \mathrm{l}$ ISTD solution $(50.0 \mathrm{ng} / \mathrm{ml}$ of AZD3293 and AZ13569724) was added, followed by the addition of $450 \mu$ of $0.1 \%$ formic acid ACN. After mixing and centrifugation, the extract was evaporated to dryness and the residue was reconstituted into $150 \mu \mathrm{l}$ ACN:water (30:70, v:v) with $0.1 \%$ formic acid and the resulting solution was kept refrigerated prior to LC-MS/MS analysis. 
Human CSF was either collected continuously into $15 \mathrm{ml}$ Falcon tubes via indwelling catheter and silicone tubing using a peristaltic pump or discretely by aspiration during the lumbar puncture procedure and placed into $15 \mathrm{ml}$ Centristar orange-cap tubes. After mixing and centrifugation, a $500 \mu \mathrm{l}$ aliquot of CSF was transferred using a calibrated pipette or syringe into a $1.8 \mathrm{ml}$ NUNC PP tube containing $5 \mu \mathrm{l}$ of $0.2 \mathrm{M}$ CHAPS solution in the clinic to prevent nonspecific binding. Samples were stored at nominal $-70^{\circ} \mathrm{C}$ in an upright position within $60 \mathrm{~min}$ of collection and kept frozen at this temperature until shipment. To each $100 \mu \mathrm{l} \mathrm{CSF}$ sample, $25 \mu \mathrm{l}$ ISTD solution $(50 \mathrm{ng} / \mathrm{ml}$ of AZD3293 and AZ13569724) was added, followed by the addition of $450 \mu \mathrm{l}$ of $0.1 \%$ formic acid ACN. After mixing and centrifugation, $400 \mu \mathrm{l}$ of extract was evaporated to dryness and the residue was reconstituted into $150 \mu \mathrm{l} \mathrm{ACN}$ :water (30:70, v:v) with 0.1\% formic acid, and the resulting solution was kept refrigerated prior to LC-MS/MS analysis.

\section{Analytical method validation}

The methods for detecting AZD3293 and AZ13569724 in plasma, urine, CSF and PUF were developed and validated based on current guidance [6,7] and laboratory standard operating procedures.

The outline of the key validation experiments conducted is described in the following sections.

\section{Accuracy \& precision}

The accuracy and precision of the methods were evaluated for each individual matrix using six replicates of QC samples at four concentrations over the calibration range tested: lower limit of quantitation (LLOQ), low concentration QC (LQC), medium concentration QC (MQC) and high concentration QC (HQC). The within- and between-batch measurements were determined in three separate analytical runs. To be considered acceptable, the within- and between-batch coefficient of variation (CV) was to be $\leq 15 \%$ (except $\leq 20 \%$ at the LLOQ). Similarly, the accuracy (reported as bias) was to be $\pm 15 \%$ of the nominal concentration (except $\pm 20 \%$ at the LLOQ). For the QC samples in an analytical run to be considered acceptable, the bias of one-half of the QC samples at each concentration and two-thirds of all the QC samples was to be within $\pm 15 \%$ of the theoretical concentration.

The reproducibility of the calibration regression model was assessed during the within- and betweenbatch accuracy. For a calibration curve to be acceptable, the back-calculated calibration concentrations needed to be within $\pm 15 \%$ of the nominal $( \pm 20 \%$ at the LLOQ), and no more than $25 \%$ of the calibration standards could be excluded from the curve.

To assess carryover in the three accuracy and precision batches, two blank matrix samples were injected after the two highest calibration samples. The analyte (and internal standard) peak area responses in the blank

\begin{tabular}{|c|c|c|c|c|c|c|c|c|c|c|}
\hline \multirow{2}{*}{$\begin{array}{l}\text { Human } \\
\text { sample } \\
\text { blank }\end{array}$} & \multirow{2}{*}{$\begin{array}{l}\text { Range } \\
\text { (ng/ml) }\end{array}$} & \multirow{2}{*}{$\begin{array}{l}\text { Calibration } \\
\text { standards } \\
(\mathrm{ng} / \mathrm{ml})\end{array}$} & \multirow{2}{*}{$\begin{array}{l}\text { Calibration } \\
\text { model }\end{array}$} & \multirow[t]{2}{*}{$\mathbf{r}^{2}$ (range) } & \multirow{2}{*}{$\begin{array}{l}\text { Matrix/sample } \\
\text { volume }(\mathrm{ml})\end{array}$} & \multicolumn{5}{|c|}{ Concentration (ng/ml) } \\
\hline & & & & & & LLOQ & LQC & MQC & HQC & DilQC \\
\hline Plasma & $0.500-500$ & $\begin{array}{l}0.500,1.00 \\
5.00,10.0 \\
50.0,100 \\
450,500\end{array}$ & $\begin{array}{l}\text { Linear } \\
\text { regression } \\
\text { and } 1 / x^{2}\end{array}$ & $0.9959-0.9991$ & 0.050 & 0.500 & 1.50 & 25.0 & 375 & $\begin{array}{l}25,00^{\ddagger} \\
(18,000)\end{array}$ \\
\hline Urine $^{\dagger}$ & $10.0-10,000$ & $\begin{array}{l}10.0,20.0 \\
100,500 \\
2000,5000 \\
9000,10,000\end{array}$ & $\begin{array}{l}\text { Linear } \\
\text { regression } \\
\text { and } 1 / x^{2}\end{array}$ & $0.9955-0.9971$ & 0.050 & 10.0 & 30.0 & 400 & 8000 & 50,000 \\
\hline $\begin{array}{l}\text { Plasma } \\
\text { ultrafiltrate }^{\dagger}\end{array}$ & $0.400-400$ & $\begin{array}{l}0.400,0.80 \\
4.00,8.00 \\
40.0,80.0 \\
360,400\end{array}$ & $\begin{array}{l}\text { Linear } \\
\text { regression } \\
\text { and } 1 / x^{2}\end{array}$ & $0.9957-0.9993$ & 0.075 & 0.400 & 1.20 & 20.0 & 300 & 2000 \\
\hline $\mathrm{CSF}^{+}$ & $0.100-100$ & $\begin{array}{l}0.100,0.200 \\
0.800,2.00 \\
10.0,50.0 \\
90.0,100\end{array}$ & $\begin{array}{l}\text { Linear } \\
\text { regression } \\
\text { and } 1 / x^{2}\end{array}$ & $0.9961-0.9987$ & 0.100 & 0.100 & 0.300 & 5.00 & 80.0 & 5000 \\
\hline
\end{tabular}


Table 2. Precision and accuracy of the quantitation of AZD3293 and AZ13569724 in quality control samples.

\begin{tabular}{|c|c|c|c|c|c|c|c|}
\hline \multirow[t]{2}{*}{ Matrix } & \multirow[t]{2}{*}{ Analyte } & \multirow[t]{2}{*}{ Parameter } & \multicolumn{5}{|c|}{ QC level } \\
\hline & & & LLOQ & LQC & MQC & HQC & DilQC \\
\hline \multirow[t]{8}{*}{ Plasma } & \multirow[t]{4}{*}{ AZD3293 } & Between-batch accuracy (bias, \%) & 2.4 & -4.0 & 0.0 & -1.1 & $0.8^{+}$ \\
\hline & & Within-batch accuracy (bias, \%) & $0.0-5.2$ & $-8.0-0.00$ & $-2.0-3.6$ & $-5.6-4.3$ & NA \\
\hline & & Between-batch precision (CV, \%) & 7.9 & 5.9 & 4.2 & 5.6 & $2.1^{\dagger}$ \\
\hline & & Within-batch precision (CV, \%) & $3.9-10.6$ & $3.1-5.8$ & $2.4-4.7$ & $3.5-4.3$ & NA \\
\hline & \multirow[t]{4}{*}{ AZ13569724 } & Between-batch accuracy (bias, \%) & -5.4 & -4.7 & -2.8 & -3.7 & $-3.6^{\dagger}$ \\
\hline & & Within-batch accuracy (bias, \%) & $-18.0-12.6$ & $-10.7-4.0$ & -5.2 to -0.4 & -5.3 to -0.8 & NA \\
\hline & & Between-batch precision (CV, \%) & 16.1 & 10.0 & 3.4 & 4.1 & $2.9^{+}$ \\
\hline & & Within-batch precision (CV, \%) & $3.2-11.8$ & $3.4-11.4$ & $2.1-3.6$ & $2.9-4.2$ & NA \\
\hline \multirow[t]{8}{*}{ Urine } & \multirow[t]{4}{*}{ AZD3293 } & Between-batch accuracy (bias, \%) & 7.0 & 5.0 & 3.3 & 2.1 & -0.2 \\
\hline & & Within-batch accuracy (bias, \%) & $2.0-11.0$ & $2.0-10.3$ & $-2.3-11.5$ & $-1.3-5.3$ & $-6.8-6.6$ \\
\hline & & Between-batch precision (CV, \%) & 9.7 & 7.2 & 7.7 & 4.2 & 7.8 \\
\hline & & Within-batch precision ( $\mathrm{CV}, \%)$ & $5.2-14.0$ & $4.3-8.3$ & $4.2-6.0$ & $3.1-3.9$ & $2.4-4.8$ \\
\hline & \multirow[t]{4}{*}{ AZ13569724 } & Between-batch accuracy (bias, \%) & -1.5 & 2.7 & 1.0 & 0.4 & -0.4 \\
\hline & & Within-batch accuracy (bias, \%) & $-7.5-7.0$ & $-4.7-10.7$ & $-4.8-8.3$ & $-5.8-6.8$ & $-7.6-7.0$ \\
\hline & & Between-batch precision (CV, \%) & 12.1 & 8.6 & 6.8 & 6.0 & 8.6 \\
\hline & & Within-batch precision (CV, \%) & $10.7-10.8$ & $4.6-8.6$ & $1.9-6.1$ & $2.1-4.2$ & $1.2-6.4$ \\
\hline \multirow[t]{8}{*}{ Plasma ultrafiltrate } & \multirow[t]{4}{*}{ AZD3293 } & Between-batch accuracy (bias, \%) & -0.8 & 5.0 & 2.5 & 1.0 & $0.5^{\dagger}$ \\
\hline & & Within-batch accuracy (bias, \%) & $-7.0-7.3$ & $1.7-10.0$ & $1.0-4.5$ & $0.0-2.0$ & NA \\
\hline & & Between-batch precision (CV, \%) & 12.4 & 6.3 & 3.0 & 3.9 & $3.3^{+}$ \\
\hline & & Within-batch precision (CV, \%) & $6.5-13.5$ & $4.0-6.8$ & $2.4-3.3$ & $3.1-5.2$ & NA \\
\hline & \multirow[t]{4}{*}{ AZ13569724 } & Between-batch accuracy (bias, \%) & 0.5 & 0.0 & 1.5 & -1.3 & $1.0^{+}$ \\
\hline & & Within-batch accuracy (bias, \%) & -2.3 to 2.3 & $-1.7-1.7$ & $-0.5-3.0$ & $-2.7-41.0$ & NA \\
\hline & & Between-batch precision (CV, \%) & 7.6 & 4.5 & 2.6 & 3.0 & $2.8^{+}$ \\
\hline & & Within-batch precision (CV, \%) & $7.2-8.9$ & $2.7-5.7$ & $1.2-2.8$ & $1.8-3.6$ & NA \\
\hline \multirow[t]{8}{*}{ CSF } & \multirow[t]{4}{*}{ AZD3293 } & Between-batch accuracy (bias, \%) & -4.1 & -1.7 & 0.4 & 1.0 & $-0.5^{\dagger}$ \\
\hline & & Within-batch accuracy (bias, \%) & $-10.9-9.0$ & $-6.7-1.3$ & $-1.0-1.4$ & $0.3-1.9$ & NA \\
\hline & & Between-batch precision (CV, \%) & 12.6 & 8.1 & 3.6 & 6.4 & $2.1^{\dagger}$ \\
\hline & & Within-batch precision (CV, \%) & $7.3-9.8$ & $4.4-10.9$ & $2.0-5.3$ & $2.7-10.7$ & NA \\
\hline & \multirow[t]{4}{*}{ AZ13569724 } & Between-batch accuracy (bias, \%) & -0.1 & -3.0 & -2.8 & 1.1 & $-2.5^{\dagger}$ \\
\hline & & Within-batch accuracy (bias, \%) & $-10.4-13.0$ & $-4.3-0.0$ & -6.0 to -1.0 & $-0.6-2.8$ & NA \\
\hline & & Between-batch precision (CV, \%) & 15.5 & 5.8 & 3.6 & 6.0 & $1.0^{+}$ \\
\hline & & Within-batch precision (CV, \%) & $8.4-15.9$ & $5.3-6.2$ & $1.8-3.6$ & $2.4-10.0$ & NA \\
\hline
\end{tabular}

matrix samples were compared with the relevant analyte area response of the lowest calibration standard representing the LLOQ of the method or, for the ISTD, the area response of the control zero sample (i.e., matrices fortified with ISTD only). The target value of the analyte peak area following the two highest concentration calibration samples was to be $\leq 20 \%$ of the correspond- ing analyte response of the LLOQ level, while the target value of the ISTD peak area was to be $\leq 5 \%$ of the ISTD response in the control zero sample.

\section{Sample dilution}

The effect of dilution was evaluated by QC samples prepared with analyte concentrations above the upper 


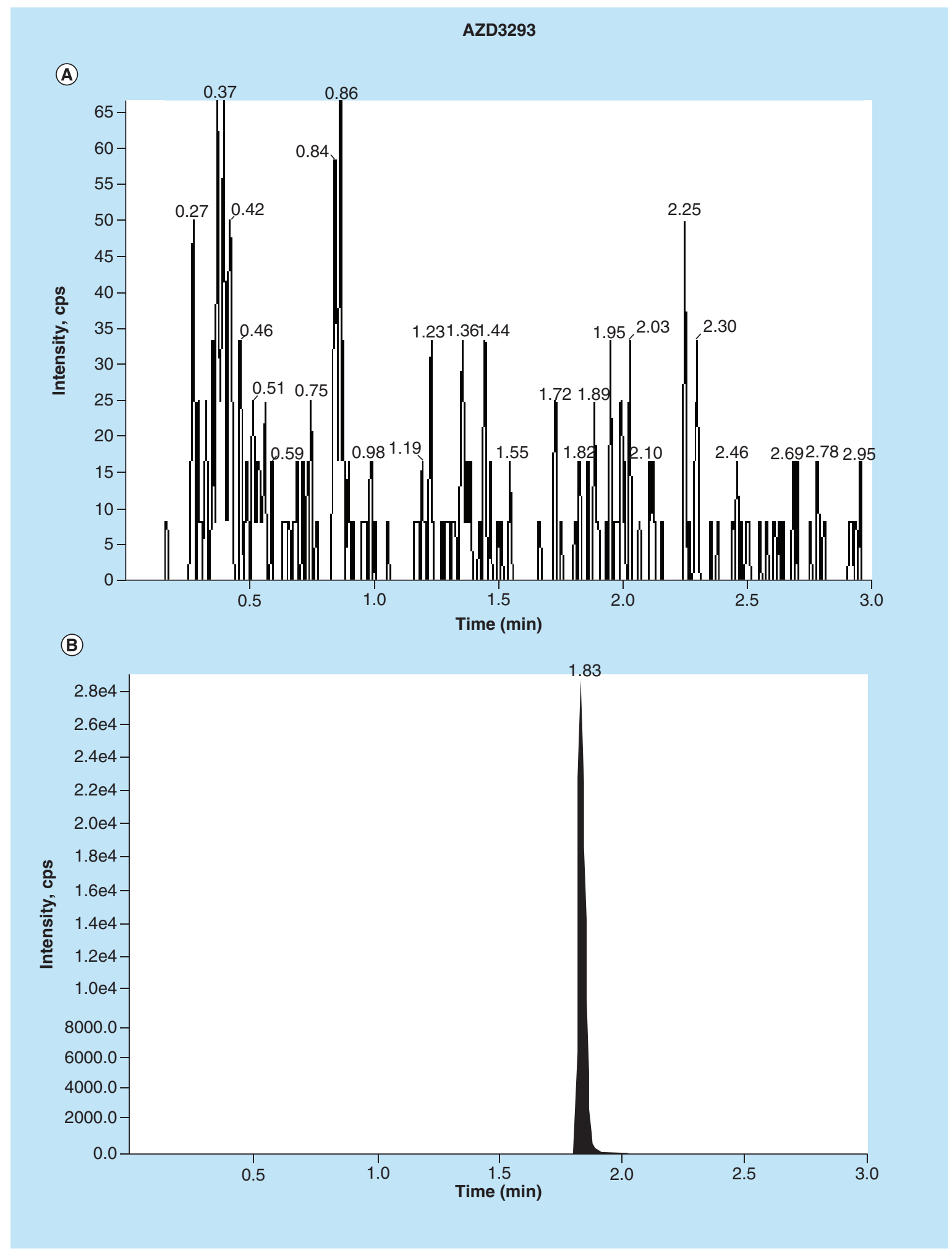




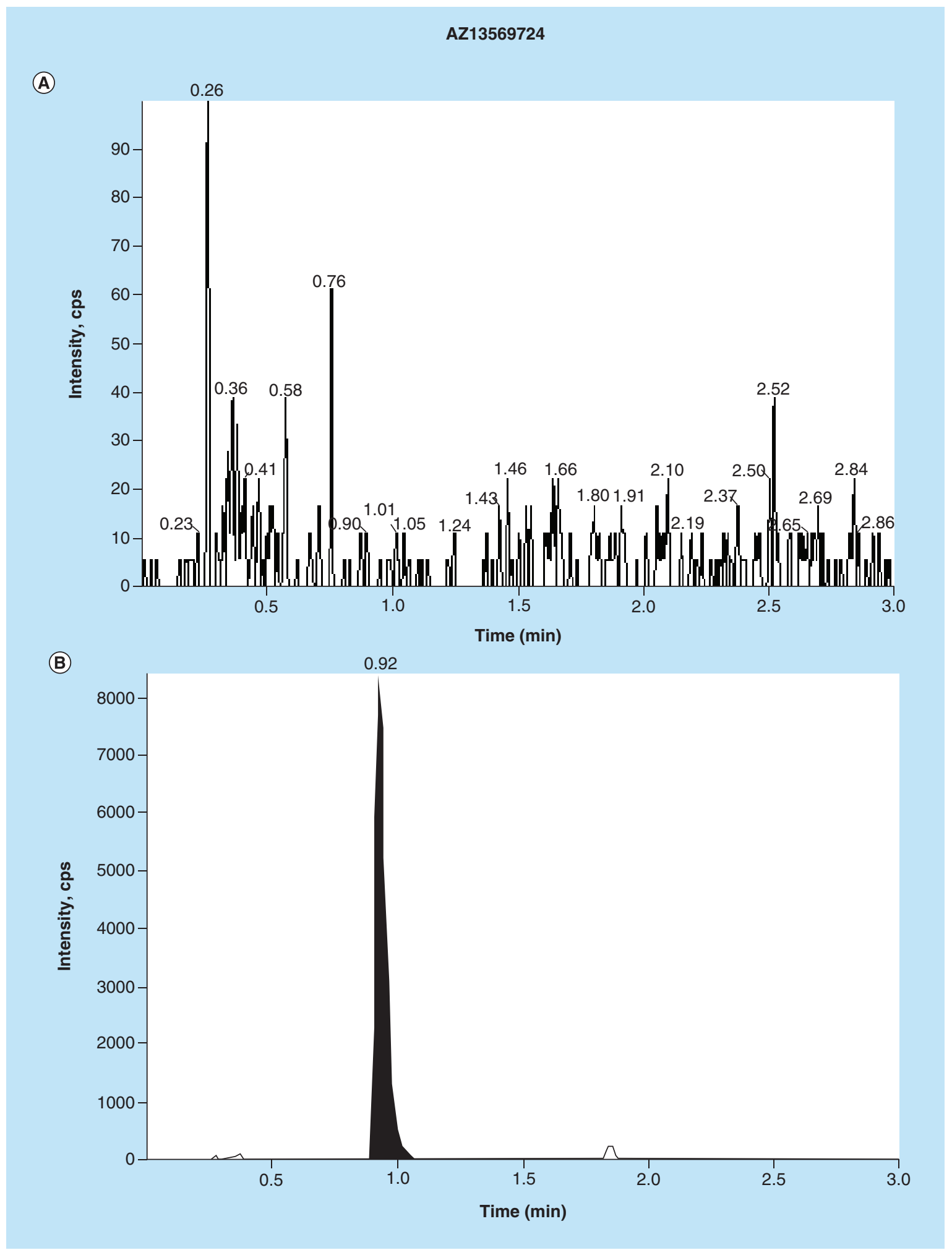




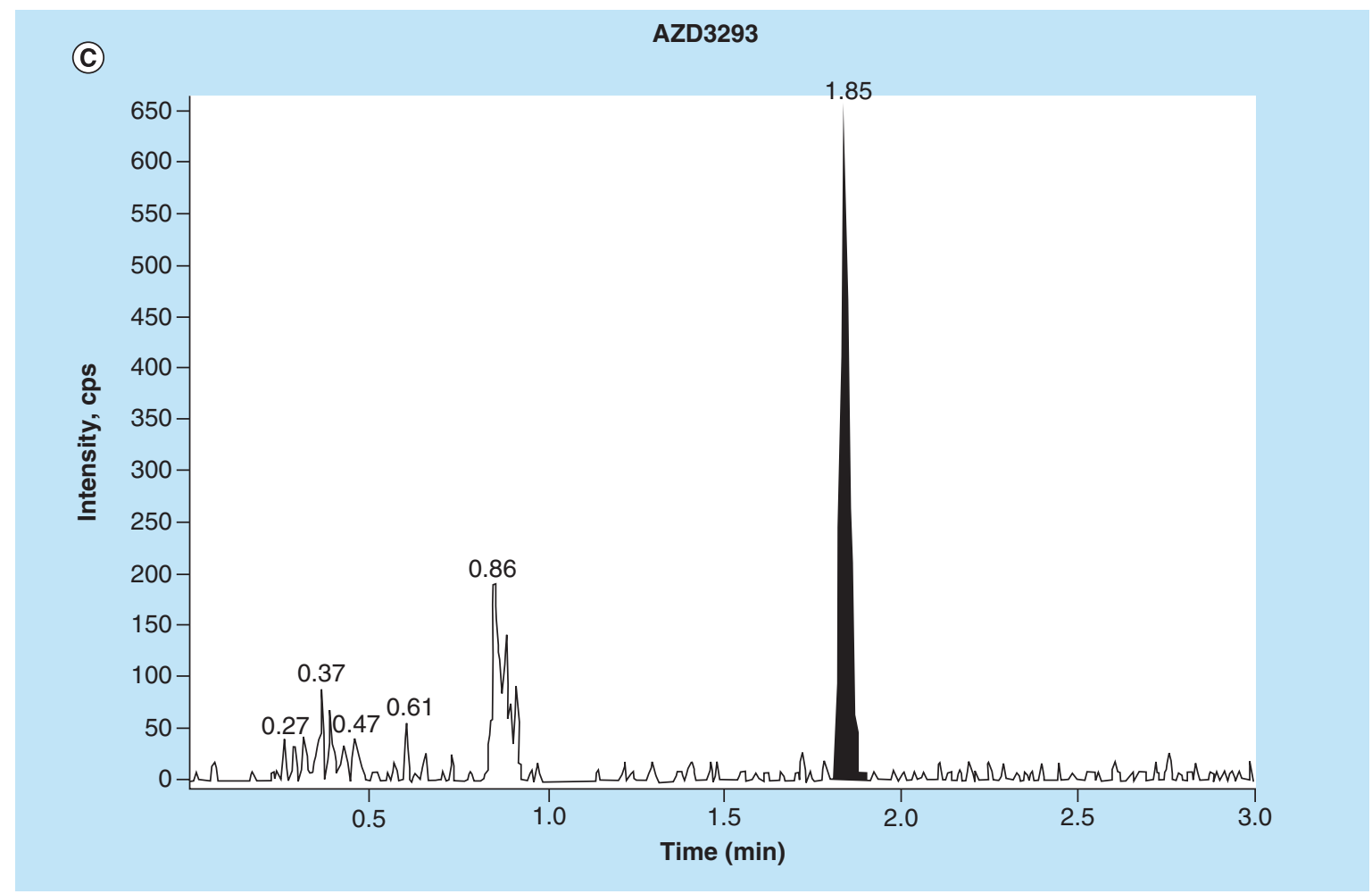

Figure 2. Analytical selectivity for the plasma method: representative chromatograms (cont. from previous left-hand page). AZD3293 (left pages) and AZ13569724 (right pages) for (A) blank human plasma, (B) internal standard alone, and (C) LLOQ $(0.500 \mathrm{ng} / \mathrm{ml})$.

LOQ of the relevant calibration curves and at a level expected to exceed predicted sample concentrations. In each case, six replicate samples were diluted with the appropriate matrix to bring levels within the calibration range. To be acceptable, the precision of the replicates was to be $\leq 15 \%(\mathrm{CV})$ and the mean bias was to be within $\pm 15 \%$ of the nominal concentration, typically a one in ten dilutions, or one in 100 dilutions for plasma.

\section{Recovery}

Three replicates were prepared at the LQC, MQC and HQC level for each of the recovery samples by adding AZD3293 and AZ13569724 (where required), plus ISTD, to the relevant extracted blank human matrix. Recoveries were expressed as percentages by comparing the mean peak area of extracted QC samples with the mean peak area of the recovery samples at the corresponding concentration. The percentage recovery values ideally were to be within a 30\% range across the QC concentrations; however, scientific judgment could be used if any trends in the data were observed.

\section{Selectivity}

The selectivity of the assay was assessed using blank matrix from six different individuals. Each individual blank matrix was analyzed and any responses in the chromatographic regions of interest assessed. The cri- terion for acceptance, defined as an interfering peak, was to be $\leq 20 \%$ of the relevant analyte peak area for the LLOQ and an ISTD interfering peak was to be $\leq 5 \%$ of the peak area for the control zero sample analyzed within the same run. Triplicates of each individual blank matrix were also spiked at the LQC level (with ISTD) and extracted and analyzed. The mean accuracy of each individual sample result was to be within $\pm 15 \%$ of the nominal concentration. Selectivity was also assessed by preparing HQC samples containing each of the individual analytes and looking for responses in the other analyte chromatographic regions.

\section{Interference tests}

To assess potential interferences that may affect the quantitation of AZD3293 and AZ13569724 in the presence of each other, six replicates of individually spiked HQC samples were prepared with AZD3293 alone or AZ13569724 alone, with internal standard (ISTD). To be acceptable, the CV had to be $\leq 15 \%$ and the mean bias had to be within $\pm 15 \%$ of the theoretical concentration. The samples were also evaluated for potential interferences at the retention times of each other and the criterion for acceptance was defined such that an interfering peak should have been less than $20 \%$ of the peak area for the LLOQ analyzed within the same run. 


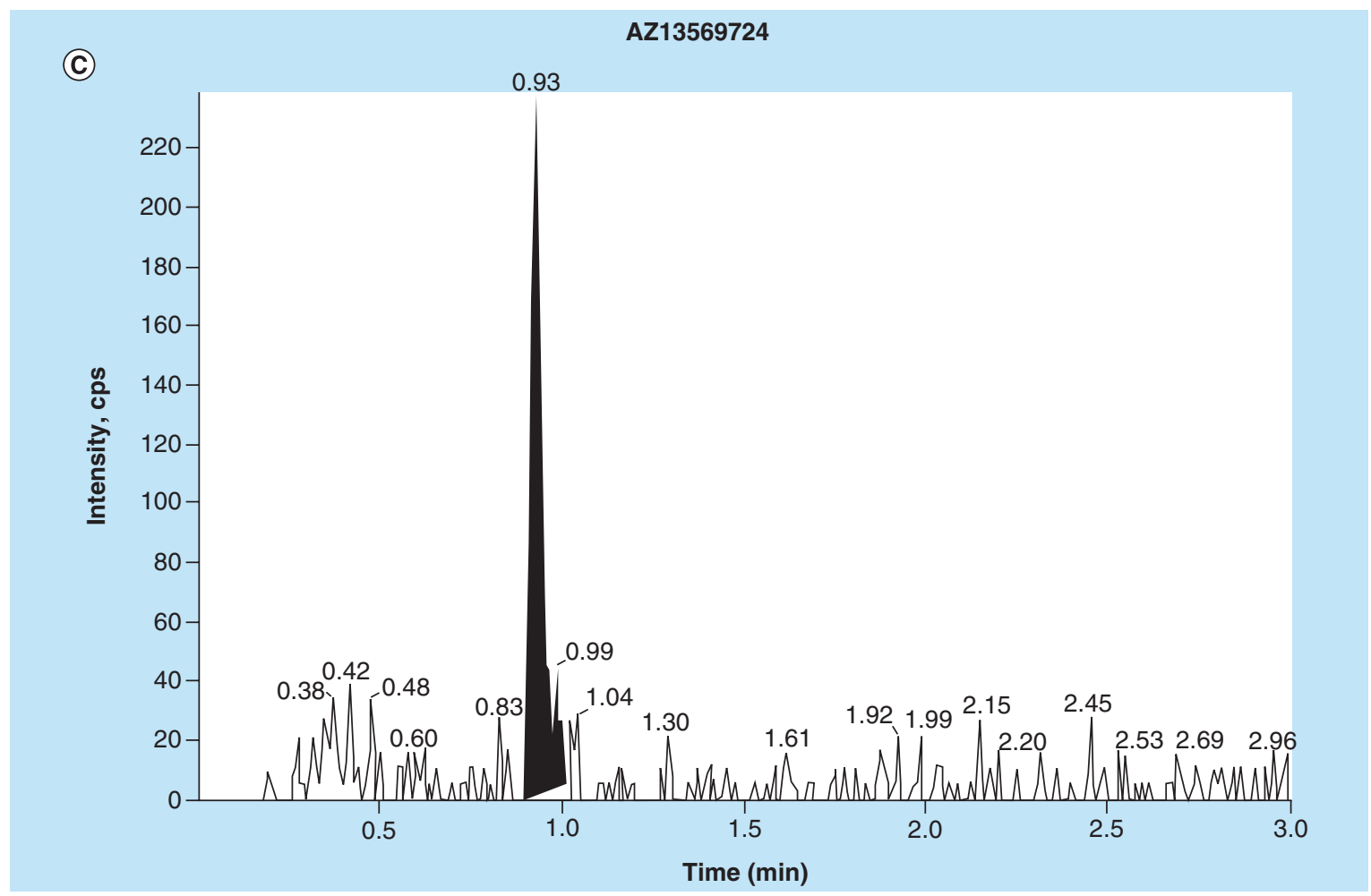

Figure 2. Analytical selectivity for the plasma method: representative chromatograms (cont. from previous right-hand page). AZD3293 (left pages) and AZ13569724 (right pages) for (A) blank human plasma, (B) internal standard alone, and (C) LLOQ $(0.500 \mathrm{ng} / \mathrm{ml})$.

\section{Matrix effects}

The relative effect of each of the biological matrices on the ionization of each analyte was assessed by using extracted blank samples from six individual biological matrix lots. For each analyte and matrix combination, the blank samples were processed and the extracts were spiked with analyte corresponding to the LQC and $\mathrm{HQC}$ analyte concentrations as well as the internal standards. In addition, three replicates of a pure solution (devoid of matrix) containing AZD3293 and AZ13569724, and ISTDs at the relevant LQC and HQC concentrations compared with the spiked extracts, assuming $100 \%$ recovery, were analyzed. The matrix factor for each of the six individuals, calculated separately for the analyte and the ISTD, was determined by the ratio between the peak area for the postextraction spike samples and the mean response of the pure solution peak areas. To be acceptable, the CV of the normalized matrix factor (analyte matrix factor over ISTD matrix factor) was to be $\leq 15 \%$.

The assessment of any impact on the plasma method of hemolyzed plasma samples was also determined by analyzing six replicates of LQC concentration samples prepared in blank human plasma containing $2 \%$ lysed whole blood. Similarly, assessment in hyperlipidemic plasma was performed by analyzing six replicates of LQC and HQC samples prepared in blank plasma with a high-lipid content $(\geq 300 \mathrm{mg} / \mathrm{dl}$ triglyceride level), which was sourced commercially. To be acceptable, the precision of the replicates was to be $\leq 15 \%(\mathrm{CV})$ and the mean bias was to be within $\pm 15 \%$ of the nominal concentration.

\section{Stability}

The stability of AZD3293 and AZ13569724 was established under a variety of storage conditions. The stability of each analyte (and corresponding ISTD stock solutions) was assessed, as well as the stability of the stored intermediate solutions.

Short- and long-term matrix stability was assessed for each analyte using six replicates at the low and high QC sample concentrations at relevant temperatures, for example, room temperature, refrigerated wet ice and frozen nominal -20 and $/$ or $-70^{\circ} \mathrm{C}$. To be acceptable, the precision of the replicates was to be $\leq 15 \%(\mathrm{CV})$ and the mean bias from the nominal concentration of QC samples was to be within $\pm 15 \%$. Freeze/thaw sample stability was also assessed in a similar manner. To support the blood (plasma) sample collection process, stability in whole blood was investigated at room temperature and on wet ice, and assessed using the mean peak area ratios of the analytes in plasma prepared after storage versus the mean peak area ratios in the reference sample response.

Processed sample viability (to allow batches to be stored before analysis, e.g., in the autosampler) and processed 
sample stability (to give an indication of the extracted sample stability) were also assessed. To be acceptable, the QCs should meet standard batch acceptance criteria.

\section{Results \& discussion}

The results and discussion described here focuses on the assay validation performances for simultaneously measuring AZD3293 and AZ13569724 in various human biological matrices. The special requirement for CSF sample collection was also evaluated and discussed. These methods have been successfully used in clinical trials and the assay performance evaluated by incurred sample reanalysis (ISR) results are also discussed.

\section{Sensitivity, precision \& accuracy}

Calibration curves for validation were produced for AZD3293 and AZ13569724 (Table 1). It was also confirmed that, where necessary, samples could be diluted into the ranges of the methods by tenfold (urine, plasma and PUF) or up to 100-fold (plasma and CSF) as assessed. The curve fit selected for the all data curves was a linear regression that required a $1 / \mathrm{x}^{2}$ concentration weighting. This performed well across the different ranges, analytes and matrices. Previous reported BACE1 compound AZD3839 [14] had to use two analytical methods in human plasma, with a low range $(0.1-100 \mathrm{nmol} / \mathrm{l})$ and a high range assay $(5-5000 \mathrm{nmol} / \mathrm{l})$, to support the single ascending dose (SAD) study.

The LLOQ for plasma was achieved at $0.5 \mathrm{ng} / \mathrm{ml}$ for both AZD3293 and AZ1569724. For PUF samples, in consideration of the in vitro protein binding data (about 7.7 and 37\% free of AZD3293 and AZ1569724 in human plasma, respectively [5]), a lower LLOQ would be needed to fully assess the PK of free drug. However, since only selected samples at $\mathrm{C}_{\max }$ were required for measurement to confirm the in vitro protein binding data, an LLOQ of $0.4 \mathrm{ng} / \mathrm{ml}$ was determined to be adequate to measure the clinical samples. As the concentration in CSF was expected to be much lower than in plasma, a fivefold lower LLOQ $(0.1 \mathrm{ng} / \mathrm{ml})$ was established in CSF for AZD3293 and AZ13569724.

The precision and accuracy of the methods were assessed in multiple analytical batches using multiple replicates of QCs (Table 2). Overall, the accuracy and precision of the methods for all the analytes and matrices met criteria. The LLOQ for each range was established with the QC replicates at this level, meeting the precision and accuracy criteria of $\pm 20 \%$. Analyte carryover was not a concern overall ( $>20 \%$ of the LLOQ calibration standard response). Specificity of the plasma method was demonstrated in samples obtained from subjects who had concomitantly received one or more of six drugs that could be potentially co-administered in future clinical studies.

High recoveries for AZD3293 and AZ13569724, and the respective ISTDs, were observed across the concentration ranges within each matrix (Supplementary Table 1). This level of recovery was considered appropriate to obtain precise and accurate quantitation of AZD3293 and AZ13569724 in the assay ranges.

\section{Selectivity, interference test \& matrix factor evaluation}

The method demonstrated acceptable selectivity from matrices for the quantitation of AZD3293 and AZ13569724 in human plasma, urine, PUF and CSF (sample chromatograms for blank plasma, internal standard only, and LLOQ in plasma are shown in Figure 2 for AZD3293 [left column] and AZ13569724 [right column]). Minimal effect of the matrix was observed, and any matrix effect was consistent and did not compromise the quantitation of AZD3293 and AZ13569724 (Supplementary Table 2). Furthermore, the presence of hemolyzed red blood cells in human plasma did not affect the quantitation of AZD3293 and AZ13569724 in the plasma method. It was also demonstrated that AZD3293 and AZ13569724 in human plasma with nat-

\begin{tabular}{|c|c|c|c|c|}
\hline \multirow{2}{*}{ Test } & \multicolumn{4}{|c|}{ AZD3293 and AZ13569724 } \\
\hline & Plasma & Urine $^{\dagger}$ & PUF $^{+}$ & $\mathrm{CSF}^{\dagger}$ \\
\hline Short term at room temperature & At least $24 \mathrm{~h}^{\ddagger}$ & At least $24 \mathrm{~h}$ & At least $24 \mathrm{~h}$ & At least $24 \mathrm{~h}^{\S}$ \\
\hline Long term at -60 to $-80^{\circ} \mathrm{C}$ and -10 to $-30^{\circ} \mathrm{C}$ & At least 365 days & At least 365 days & At least 94 days & At least 92 days" \\
\hline Processed sample viability at $2-8^{\circ} \mathrm{C}$ & At least $139 \mathrm{~h}$ & At least $118 \mathrm{~h}$ & At least $139 \mathrm{~h}$ & At least $115 \mathrm{~h}$ \\
\hline $\begin{array}{l}\text { Freeze/thaw stability at }-60 \text { to }-80^{\circ} \mathrm{C} \text { and } \\
-10 \text { to }-30^{\circ} \mathrm{C}\end{array}$ & At least five-times & At least five-times & At least three-times & At least five-times" \\
\hline $\begin{array}{l}\text { With CHAPS added. } \\
\text { "Whole blood stability: at least } 2 \mathrm{~h} \text { at room temperature } \\
\text { sStability in human CSF without CHAPS: at least } 30 \text { min } \\
\text { "Only evaluated at }-60 \text { to }-80^{\circ} \mathrm{C} \text {. }\end{array}$ & $\begin{array}{l}\text { d on wet ice. } \\
\text { both analytes. }\end{array}$ & & & \\
\hline
\end{tabular}


Table 4. Evaluation of AZD3293 and AZ13569724 in human cerebrospinal fluid sample collection device.

\begin{tabular}{|c|c|c|c|c|c|c|c|}
\hline \multirow[t]{2}{*}{ Condition } & \multirow[t]{2}{*}{ Parameter } & \multicolumn{3}{|c|}{ AZD3293 } & \multicolumn{3}{|c|}{ AZ13569724 } \\
\hline & & $\begin{array}{l}\text { QC-L } \\
(0.300 \mathrm{ng} / \mathrm{ml})\end{array}$ & $\begin{array}{l}\text { QC-M } \\
(5.00 \mathrm{ng} / \mathrm{ml})\end{array}$ & $\begin{array}{l}\text { QC-H } \\
(80.0 \mathrm{ng} / \mathrm{ml})\end{array}$ & $\begin{array}{l}\text { QC-L } \\
(0.300 \mathrm{ng} / \mathrm{ml})\end{array}$ & $\begin{array}{l}\text { QC-M } \\
(5.00 \mathrm{ng} / \mathrm{ml})\end{array}$ & $\begin{array}{l}\text { QC-H } \\
(80.0 \mathrm{ng} / \mathrm{ml})\end{array}$ \\
\hline \multirow[t]{3}{*}{ Set-up 1} & Mean & 0.290 & 4.92 & 79.6 & 0.285 & 4.91 & 79.1 \\
\hline & CV $(\%)$ & 1.1 & 4.2 & 1.6 & 3.4 & 2.0 & 0.6 \\
\hline & Bias (\%) & -3.3 & -1.6 & -0.5 & -5.0 & -1.8 & -1.1 \\
\hline \multirow[t]{4}{*}{ Set-up 2} & Mean & 0.280 & 4.90 & 84.9 & 0.306 & 4.80 & 81.7 \\
\hline & CV $(\%)$ & 1.7 & 0.6 & 1.5 & 2.1 & 0.1 & 2.1 \\
\hline & Bias (\%) & -6.7 & -2.0 & 5.1 & 2.0 & -4.0 & 2.1 \\
\hline & $\begin{array}{l}\% \text { Change vs } \\
\text { Set-up } 1\end{array}$ & -3.4 & -0.4 & 5.7 & 7.4 & -2.2 & 3.3 \\
\hline \multirow[t]{4}{*}{ Set-up 3} & Mean & 0.198 & 3.57 & 63.4 & 0.284 & 4.95 & 80.5 \\
\hline & CV $(\%)$ & 6.7 & 1.0 & 1.3 & 4.9 & 1.6 & 3.0 \\
\hline & Bias (\%) & -34.0 & -28.6 & -20.8 & -5.3 & -1.0 & 0.6 \\
\hline & $\begin{array}{l}\% \text { Change vs } \\
\text { Set-up } 1\end{array}$ & -29.3 & -27.1 & -24.6 & -7.2 & 3.1 & -1.5 \\
\hline $\begin{array}{l}\text { Set-up 1: Store } \\
\text { Set-up 2: Store } \\
\text { Set-up 3: Store } \\
\text { CHAPS: 3-[(3-- } \\
\text { medium. }\end{array}$ & $\begin{array}{l}\text { for } 30 \mathrm{~min} \text { at room } \\
\text { for } 30 \mathrm{~min} \text { at room } \\
\text { in collection tube } \\
\text { olamidopropyl) din }\end{array}$ & $\begin{array}{l}\text { erature with CHAPS } \\
\text { erature without CHA } \\
\text { t CHAPS at room te } \\
\text { ammonio]-1-propan }\end{array}$ & $\begin{array}{l}\text { in a vial. } \\
\text { APS present in a via } \\
\text { mperature for } 30 \mathrm{n} \\
\text { esulfonate hydrate }\end{array}$ & $\begin{array}{l}\text { then CHAPS was } \\
\text {, then transferre } \\
\mathrm{C}-\mathrm{H} \text { : Quality con }\end{array}$ & $\begin{array}{l}\text { d for analysis. } \\
\text { vial and CHAPS wa } \\
\text { high; QC-L: Quality }\end{array}$ & $\begin{array}{l}\text { s added for analysi } \\
\text { ontrol low; QC-M: }\end{array}$ & uality control \\
\hline
\end{tabular}

urally high lipid content did not affect the quantitation of AZD3293 and AZ13569724 in this assay.

In the assessment of interference between AZD3293 and AZ13569724 to each other in various matrices, small peaks (ranges from 28 to $60 \%$ of LLOQ) were detected in the AZ13569724 channel of the HQC samples fortified with only AZD3293 (urine, CSF and PUF). We suspect this was due to an impurity in the reference standard material. As this represented less than $0.1 \%$ of the ULOQ level and did not indicate a significant level of contribution when the two analytes were added in equal concentrations as it was done in the calibration and QC samples, this interference peak was determined to have no impact on the accuracy of the methods overall.

\section{Stability}

The stability of AZD3293 and AZ13569724 was investigated in a variety of matrices and under a variety of conditions. Both analytes demonstrated good stability under controlled conditions. The stability of stock solutions at $4{ }^{\circ} \mathrm{C}$ was known to be at least 98 days for AZD3293 and 45 days for AZ13569724 in ACN/water solution (30:70, v:v). Acceptable stability of AZD3293 and AZ13569724 was demonstrated in all human biological matrices tested at room temperature, on wet ice, during freeze-thaw cycling, in processed samples and in storage at nominal -20 and $-70^{\circ} \mathrm{C}$ (Table 3).

\section{Evaluation of adsorption in CSF collection apparatus}

For CSF collection in clinical trial, as there is minimal protein present in the CSF fluid, it is important to evaluate the potential for nonspecific binding to the collection apparatus [14]. To assess the stability and potential loss of AZD3293 and AZ13569724 through nonspecific binding during the continuous collection of human CSF via silicone tubing used in AZD3293 multiple ascending dose (MAD) study [13], three fresh human CSF pools were prepared and were spiked at three concentrations $(0.3,5$ and $80 \mathrm{ng} / \mathrm{ml})$. These pools were each gently mixed and divided into three separate portions. One portion (Set-up 1) was stored in a vial with the appropriate 1:100 ratio of CHAPS added and was used as the reference for this test. A second portion (Set-up 2) was stored in a vial with no CHAPS added and was used to assess the stability of the analytes in CSF with no CHAPS added. A third portion (Set-up 3) was stored in a collection kit with tubing (obtained from clinic site) and was used to evaluate the potential loss of AZD3293 and AZ13569724 due to nonspecific binding (adsorption) to the collection apparatus. The CSF was stored in each configuration for $30 \mathrm{~min}$ at room temperature. Following the $30 \mathrm{~min}$ storage, CHAPS was added to the vial in Set-up 2 and was analyzed. For Set-up 3, CSF was pipetted into a vial, the appropriate 1:100 ratio of CHAPS was added and then the sample was analyzed. The stability or loss of analyte concentrations 
was assessed by comparing the mean AZD3293 and AZ13569724 concentrations found for Set-up 2 to those for Set-up 1 (effect of CHAPS), and by comparing those for Set-up 3 to those for Set-up 2 (effect of storage in the collection apparatus).

Results showed that both AZD3293 and AZ13569724 were stable in human CSF for at least $30 \mathrm{~min}$ when stored at room temperature in a vial that did not contain CHAPS (Set-up 2; Table 4). Results for Set-up 1 (CHAPS added during $30 \mathrm{~min}$ storage period) were comparable to those for Setup 2, indicating that the addition of CHAPS per se does not directly affect analyte concentrations. Finally, comparison of Set-up 3 (storage in CSF collection apparatus) with Set-up 1 showed that while there was no significant loss of AZ13569724, modest adsorption was noted for AZD3293 across all three concentrations (range 24-29\%). These data have bearing on our understanding of the relationship of exposures observed in plasma and CSF relative to PD effects [13].

\section{Assay performance in clinical studies \& ISR}

As the QC samples used during validation may not mimic clinical study samples, ISR of study samples is recommended [6-7,15]. To confirm acceptable performance of the method in the study, it is recommended in the Covance-AstraZeneca Clinical Bioanalysis Alliance that ISR is conducted in all clinical studies for plasma and, at a minimum, in the first study using the method in a new matrix or where there may be significant population differences.
For the analytical methods described in this paper, ISR has been conducted in plasma for all the studies and in urine and CSF when these methods were used for the first time. ISR evaluation was not conducted for PUF samples due to the low overall sample numbers and based on the fact that such results were generally not central to PK parameter calculations or analyses. As per recommended guidelines, at least $10 \%$ of samples were analyzed for ISR where the total sample number is $\leq 1000$; above this, an additional $5 \%$ samples are added and representative clinical samples across the analyte concentration range were selected. The concentration obtained for the initial analysis and the concentration obtained by reanalysis should be within $20 \%$ of the mean for at least $67 \%$ of the samples.

The validated methods have been used to support AZD3293 single and multiple ascending dose studies (SAD and MAD) in healthy subjects (both Caucasian and Japanese) as well as a small number of mild-to-moderate AD patients [13]. In addition, a number of clinical pharmacology studies were conducted using the plasma and/or urine method. In all these studies, calibration standard data, QC sample data, ISR data and chromatograms indicate that these methods performed acceptably during the sample analysis phase.

A total of over 6000 plasma samples were analyzed and reported in the seven Phase I clinical studies noted above, and approximately 550 ISR plasma samples were evaluated (Table 5). In all cases, the results of ISR evaluation indicated that overall the methods per-

Table 5. Incurred sample re-analysis in clinical studies.

\begin{tabular}{|c|c|c|c|c|}
\hline Study & $\begin{array}{l}\text { Number of plasma } \\
\text { samples }\end{array}$ & $\begin{array}{l}\text { ISR (plasma): } \\
\text { number of samples }\end{array}$ & $\begin{array}{l}\text { ISR pass rate (\%) } \\
\text { AZD3293 }\end{array}$ & $\begin{array}{l}\text { ISR pass rate (\%) } \\
\text { AZ13569724 }\end{array}$ \\
\hline $\begin{array}{l}\text { D5010C00001 } \\
\text { (SAD) }\end{array}$ & 1136 & 103 & 91 & 86 \\
\hline $\begin{array}{l}\text { D5010C00002 } \\
\text { (MAD) }\end{array}$ & 932 & 93 & 99 & 99 \\
\hline $\begin{array}{l}\text { D5010C00003 } \\
\text { (JSMAD) }\end{array}$ & 614 & 64 & 98 & 95 \\
\hline $\begin{array}{l}\text { D5010C00004 } \\
\text { (DDI) }\end{array}$ & 954 & 101 & 99 & 92 \\
\hline $\begin{array}{l}\text { D5010C00005 } \\
\text { (RelBio) }\end{array}$ & 660 & 66 & 100 & 97 \\
\hline $\begin{array}{l}\text { D5010C00007 } \\
\text { (human ADME) }\end{array}$ & 217 & 19 & 100 & 100 \\
\hline $\begin{array}{l}\text { D5010C00008 } \\
\text { (thorough QT) }\end{array}$ & 1639 & 102 & 100 & 100 \\
\hline
\end{tabular}


formed acceptably during the sample analysis phases in all the studies/matrices evaluated.

\section{Conclusion}

There are a number of BACE1 inhibitor drug candidates currently in clinical trials for $\mathrm{AD}$ [4], including AZD3293, which is presently in Phase III trials. This is the first time that we have reported the full details of the LC-MS/MS methods developed in human biological matrices (i.e., plasma, urine, PUF, and CSF) for AZD3293 and its major metabolite AZ13569724, which is pharmacologically active at BACE1 though less potent than the parent compound. These methods were adequately sensitive, selective, and extremely robust for the analysis of clinical samples. The reproducibility of the study data has been demonstrated through successful ISR. The full validation method established in CSF coupled with an assessment of nonspecific binding to CSF collection tubing has enabled a more fulsome understanding of the human PK/PD relationship for AZD3293. The accurate measurement of AZ13569724 in human samples facilitates the evaluation of metabolite safety for this program. The concentration data generated by these methods were instrumental in understanding human PK and selecting the dose and regimen for the Phase III studies of AZD3293 in AD (AMARANTH, NCT02245737; and DAYBREAK-ALZ, NCT02783573).

\section{Future perspective}

The availability of validated bioanalytical methods in multiple human biological matrices to simultaneously measure the AZD3293 and its metabolite AZ13569724 (M1) enables the characterization of PK properties of AZD3293 and AZ13569724 in both healthy subjects and patients, which will enable a deeper understanding of $\mathrm{PK} / \mathrm{PD}$ relationships. These methods have been used for the accurate and robust determination of AZD3293 and AZ13569724 concentrations in a number of clinical studies, and the plasma and CSF methods are currently being used to support on-going Phase III trials in patients with AD (AMARANTH, NCT02245737; and DAYBREAK-ALZ, NCT02783573).

\section{Supplementary data}

To view the supplementary data that accompany this paper, please visit the journal website at: www.future-science.com/ doi/full/10.4155/bio-2017-0003

\section{Acknowledgements}

The authors wish to acknowledge AZD3293 team members within AstraZeneca and Eli Lilly who participated in methods development or validation activities and/or contributed constructive reviews of this manuscript, and EW Ingersoll, who provided medical writing and editing services in the development of this manuscript through contract with AstraZeneca.

\section{Financial \& competing interests disclosure}

This research was funded by AstraZeneca. $\mathrm{Y} L \mathrm{~L}$ is an employee of AstraZeneca and holds AstraZeneca stock. PH Severin, MR Hoffmann and DL Miller are current employees of Covance Laboratories, Inc., the company that developed and validated the methodologies in partnership with AstraZeneca and with funding from AstraZeneca. AR Kugler is a former employee of AstraZeneca and holds AstraZeneca stock. SA Monk is an employee of Eli Lilly and Co., and holds Eli Lilly stock. The authors have no other relevant affiliations or financial involvement with any organization or entity with a financial interest in or financial conflict with the subject matter or materials discussed in the manuscript apart from those disclosed.

\section{Summary points}

- AZD3293 is a novel, potent, brain-permeable inhibitor of human BACE1. It is being developed as a potentially disease-modifying treatment for Alzheimer's disease (AD) in patients diagnosed with early $A D$, defined as the continuum of patients with mild cognitive impairment due to $A D$ and patients diagnosed with mild dementia of the Alzheimer's type.

- To support the development of AZD3293, sensitive and robust bioanalytical methods were required to quantitate the drug and its metabolite AZ13569724 (M1) in human biological samples.

- LC-MS/MS multi-analyte methods for accurate determination of AZD3293 and AZ13569724 in various human biological samples (plasma, urine, plasma ultrafiltrate and cerebrospinal fluid) were developed and validated with acceptable levels of precision, accuracy and recovery, as well as minimal matrix effect and adequate selectivity.

- Acceptable stability of AZD3293 and AZ13569724 was demonstrated in all human biological matrices tested at room temperature, on wet ice, during freeze-thaw cycling, in processed samples, and in storage at -20 and $-70^{\circ} \mathrm{C}$.

- Evaluation of nonspecific binding in CSF collection apparati showed a 27\% decrease in AZD3293 concentrations, and this has been considered when interpreting CSF AZD3293 concentrations obtained from clinical studies.

- Satisfactory ISR performances from clinical trials demonstrated the robustness of these methods. 
The medical writing support for this paper was funded by AstraZeneca.

\section{Ethical conduct of research}

The authors state that they have obtained appropriate institutional review board approval or have followed the principles outlined in the Declaration of Helsinki for all human or animal experimental investigations. In addition, for investigations in-

\section{References}

Papers of special note have been highlighted as: $\bullet$ of interest; •• of considerable interest

1 Yan R. Stepping closer to treating Alzheimer's disease patients with BACE1 inhibitor drugs. Transl. Neurodegener. 5, 13 (2016).

2 Menting KW, Claassen JA. $\beta$-secretase inhibitor; a promising novel therapeutic drug in Alzheimer's disease. Front. Aging Neurosci. 6, 165 (2014).

3 Vassar R, Kuhn PH, Haass C et al. Function, therapeutic potential and cell biology of BACE proteases: current status and future prospects. J. Neurochem. 130(1), 4-28 (2014).

4 Vassar R. BACE1 inhibitor drugs in clinical trials for Alzheimer's disease. Alzheimers Res. Ther. 6(9), 89 (2014).

- Definitive review of BACE1 inhibition and drug candidates.

5 Eketjall S, Janson J, Kaspersson K et al. AZD3293: a novel, orally active BACE1 inhibitor with high potency and permeability and markedly slow off-rate kinetics. J. Alzheimers Dis. 50(4), 1109-1123 (2016).

-• Description of preclinical pharmacology and safety data for AZD3293 and AZ13569724.

6 Guideline on Bioanalytical Method Validation. www.ema.europa.eu/docs/en_GB/document_library/

- A guideline that defines key elements necessary for the validation of bioanalytical methods.

7 Guidance for Industry: Bioanalytical Method Validation. www.fda.gov/downloads/Drugs/GuidanceCompliance

- A guideline that defines key elements necessary for the validation of bioanalytical methods.

8 Guidance for Industry: Bioanalytical Method Validation (draft guidance). www.fda.gov/downloads/drugs/guidancecompliance

-• A guideline that defines key elements necessary for the validation of bioanalytical methods. volving human subjects, informed consent has been obtained from the participants involved.

\section{Open access}

This work is licensed under the Attribution-NonCommercialNoDerivatives 4.0 Unported License. To view a copy of this license, visit http://creativecommons.org/licenses/by-nc$\mathrm{nd} / 4.0 /$

9 Ponnayyan Sulochana S, Sharma K, Mullangi R, Sukumaran SK. Review of the validated HPLC and LC-MS/MS methods for determination of drugs used in clinical practice for Alzheimer's disease. Biomed. Chromatogr. 28(11), 1431-1490 (2014).

10 Guidance for Industry: Safety Testing of Drug Metabolites. www.fda.gov/OHRMS

- Describes key guidelines for the evaluation of metabolites in safety testing.

11 Frederick CB, Obach RS. Metabolites in safety testing: "MIST" for the clinical pharmacologist. Clin. Pharmacol. Ther. 87(3), 345-350 (2010).

- Describes key guidelines for the evaluation of metabolites in safety testing.

12 Teunissen CE, Petzold A, Bennett JL et al. A consensus protocol for the standardization of cerebrospinal fluid collection and biobanking. Neurology 73(22), 1914-1922 (2009).

13 Cebers G, Alexander RC, Haeberlein SB et al. AZD3293: pharmacokinetic and pharmacodynamic effects in healthy subjects and patients with Alzheimer's disease. J. Alzheimers Dis. 55(3), 1039-1053 (2017).

-• Describes the first clinical studies of AZD3293 including pharmacokinetics following single and multiple doses.

14 Wagdy AM, Kim JC, Kim GE, Wu H, El-Shourbagy T. Effect of sample collection tubing type used in a clinical study on quantitation of pharmaceutical compounds in CSF by LC-MS/MS. Bioanalysis 3(2), 167-179 (2011).

15 Booth B, Arnold ME, Desilva B et al. Workshop report: Crystal City V - quantitative bioanalytical method validation and implementation: the 2013 revised FDA guidance. AAPS J. 17(2), 277-288 (2015).

- This is a white paper that defines key elements necessary for the validation of bioanalytical methods 